\title{
ESTADO NUTRICIONAL DE MENORES DE 5 ANOS DE IDADE EM SOBRAL-CE
}

NUTRITIONAL STATUS OF CHILDREN UNDER 5 YEARS OF AGE IN SOBRAL, CEARÁ, BRAZIL

ESTADO NUTRICIONAL DE MENORES DE 5 AÑOS DE EDAD EN SOBRAL, CEARÁ, BRASIL

Beatriz Mendes Alves João Vitor Souza Marques 2 Cynara Carvalho Parente ${ }^{3}$ Marcos Vinícius Souza Marques 4 Francisco Plácido Nogueira Arcanjo ${ }^{5}$ Karyne Gomes Cajazeiras 6

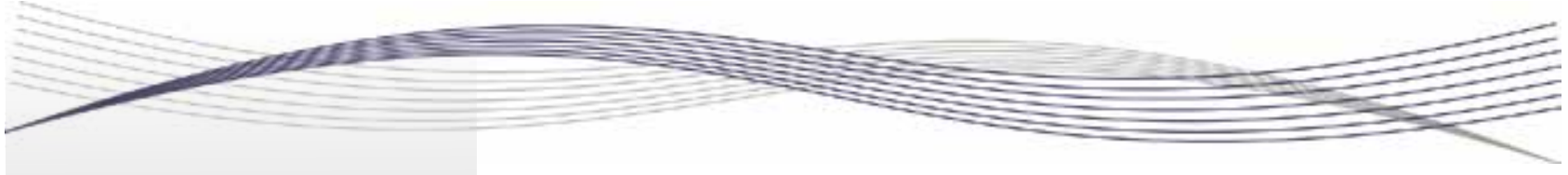

Palavras-chave:

Estado Nutricional; Criança; Atenção Primária à Saúde.

Keywords: Nutritional Status; Child; Primary Health Care.

Palabras clave: Estado Nutricional; Niño; Atención Primaria de Salud.

Submetido: $14 / 01 / 2019$

\section{RESUMO}

Este estudo teve por objetivo avaliar o estado nutricional de menores de 5 anos de idade em Sobral-CE, no período entre 2013 e 2017. Para tanto, foram usados dados dos seguintes indices nutricionais disponiveis no Sistema de Vigilância Alimentar e Nutricional do Ministério da Saúde (SISVAN/MS): a) peso-para-altura; b) peso-para-idade; c) idade-para-altura; e d) IMC-paraidade. Nesse período, constatou-se que o número de crianças acometidas por magreza e magreza acentuada aumentou no município, porém, o agravo nutricional com crescimento mais acentuado foi o sobrepeso (aumento de $58,2 \%)$. Constatou-se, ainda, aumento do número de crianças com altura baixa e muito baixa para a idade, além do peso baixo e muito baixo. 0 IMC-para-idade indicou aumento do sobrepeso e da obesidade. Diante do aumento tanto da magreza e magreza acentuada quanto do sobrepeso e da obesidade entre crianças menores de 5 anos, concluiu-se que coexistem em Sobral diversos agravos nutricionais nessa população. Portanto, mostra-se necessário adotar ações na atenção primária à saúde (APS) para reduzir e corrigir as carências nutricionais, além de investir em pesquisas voltadas à promoção de hábitos saudáveis desde a mais tenra infância.
\end{abstract}

Aprovado: $14 / 05 / 2019$

Autor(a) para Correspondência: Beatriz Mendes Alves Av. Dr. Guarani, 307, apto. 104, Derby Clube - Sobral (CE) CEP: 62042-030. E-mail: beatriz_mendes.a@ outlook.com

\footnotetext{
1. Aluna de Graduação em Medicina na Universidade Federal do Ceará (UFC). Sobral (CE), Brasil.

2. Aluno de Graduação em Medicina na UFC. Sobral (CE), Brasil.

3. Professora no Curso de Graduação em Medicina da UFC. Sobral (CE), Brasil.

4. Aluno de Graduação em Medicina na UFC. Sobral (CE), Brasil.

5. Professor no Curso de Graduação em Medicina da UFC. Sobral (CE), Brasil.

6. Aluna de Graduação em Medicina na UFC. Sobral (CE), Brasil.
} 


\section{ABSTRACT}

This study aimed to assess the nutritional status of children under 5 years of age in Sobral, Ceará, Brazil, within the period from 2013 to 2017. To do so, data from the following nutritional indexes available on the Food and Nutrition Surveillance System of the Brazilian Ministry of Health (Sistema de Vigilancia Alimentar e Nutricional do Ministério da Saúde - SISVAN/MS) were used: a) weight-for-height; b) weight-for-age; c) age-for-height; and d) BMI-for-age. Within this period, it was found that the number of children affected by thinness and marked thinness increased in the municipality, but overweight (a 58.2\% increase) was the most significant nutritional issue. An increased number of children with low height and very low height for age was also observed, in addition to low weight and very low weight. The BMI-for-age indicated an increase in overweight and obesity. Given the increase in both thinness and marked thinness, as well as overweight and obesity, among children under 5 years of age, it was concluded that many nutritional issues coexist in Sobral in this population. Therefore, it is necessary to adopt primary health care (PHC) actions to reduce and correct nutritional deficiencies, besides investing in research aimed at promoting healthy habits from the earliest childhood.

\section{RESUMEN}

Este estudio tuvo como objetivo evaluar el estado nutricional de menores de 5 años de edad en Sobral, Ceará, Brasil, en el período de 2013 a 2017. Para ello, datos de los siguientes índices nutricionales disponibles en el Sistema de Vigilancia Alimentaria y Nutricional del Ministerio de Salud de Brasil (Sistema de Vigilância Alimentar e Nutricional do Ministério da Saúde - SISVAN/MS): a) peso-para-altura; b) peso-para-edad; c) edad-para-altura; y d) IMC-para-edad. Dentro de este período, se constató que el número de niños afectados por delgadez y delgadez marcada aumentó en el municipio, pero el sobrepeso (aumento de 58,2\%) fue el problema nutricional más importante. También se constató un aumento del número de niños con altura baja y muy baja para la edad, además de peso bajo y peso muy bajo. El IMC-para-edad indicó aumento del sobrepeso y de la obesidad. Dado el aumento tanto de la delgadez y de la delgadez marcada como del sobrepeso y de la obesidad entre niños menores de 5 años, se concluyó que en Sobral coexisten muchos problemas nutricionales en esta población. Por lo tanto, es necesario adoptar acciones de atención primaria de salud (APS) para reducir y corregir las deficiencias nutricionales, además de invertir en investigaciones dirigidas a promover hábitos saludables desde la más tierna infancia.

\section{INTRODUÇÃ O}

0 Brasil vem passando por uma transição nutricional, evidenciada pelo aumento da prevalência de obesidade e sobrepeso em crianças e, em paralelo, pela redução da desnutrição infantil. As carências nutricionais se relacionam a fatores socioeconômicos; por outro lado, o sobrepeso e a obesidade refletem maior disponibilidade de alimentos processados industrialmente, com elevado teor de gorduras e açúcares, acarretando inadequado aumento de peso das crianças ${ }^{1}$.

Por dependerem da situação socioeconômica e cultural das crianças, a desnutrição e a obesidade devem ser avaliadas na atenção primária à saúde (APS), visto que é no âmbito da Política Nacional de Atenção Básica (PNAB) que se encontra a Política Nacional de Alimentação e Nutrição (PNAN) aprovada em 1999, com o intuito de desenvolver ações para respeitar, proteger, promover e prover os direitos humanos à saúde e à alimentação².

0 Sistema de Vigilância Alimentar e Nutricional do Ministério da Saúde (SISVAN/MS) disponibiliza relatórios em formato eletrônico, contendo dados como medidas antropométricas e consumo alimentar, para a avaliação nutricional nos serviços de saúde da APS. Ademais, apresenta informações gerais, como faixa etária, sexo, escolaridade, raça e cor, que possibilitam a visualização dos grupos de pacientes com obesidade, sobrepeso e desnutrição ${ }^{3}$.

A desnutrição é uma condição de alta letalidade, decorrente de inúmeros fatores, que leva à adaptação do organismo por meio de mudanças fisiológicas para tentar suprir a falta de nutrientes. Desigualdades sociais, saúde precária e moradias de difícil acesso estão entre os principais aspectos que impactam o estado nutricional dos indivíduos ${ }^{4,5}$.

0 estado nutricional materno pode acarretar agravos alimentares ao(s) filho(s), pois há associação significativa entre a desnutrição da mãe e a desnutrição aguda global da(s) criança(s) ${ }^{6}$. Para combater e prevenir a desnutrição infantil, fazse necessária uma intervenção nutricional voltada não só às crianças desnutridas, mas, também, às 
mulheres em período reprodutivo e às gestantes.

De acordo com a Organização das Nações Unidas para a Alimentação e a Agricultura (Food and Agriculture Organization of the United Nations FA0), no Brasil, a subnutrição caiu de $4,6 \%$ para menos de 2,5\%, comparando o triênio 2004-2006 ao triênio 2015-20177. Segundo dados do SISVAN/MS, em 2017, o Ceará tinha 6.904 crianças com baixo peso para a idade - esse número correspondia a $2,4 \%$ da população infantil do estado com essa condição ${ }^{3}$.

No Município de Sobral, a desnutrição também vem apresentando declínio. Provavelmente, essa queda se deve a melhorias na qualidade de vida da população, bem como à implantação de programas de suplementação alimentar ${ }^{8}$.

A avaliação do estado nutricional de crianças de 0 a 5 anos de idade, recomendada pela Organização Mundial da Saúde (OMS), deve ser realizada por meio dos dados de peso/estatura, estatura/idade, índice de massa corpórea (IMC)/idade e peso/idade e o tratamento recomendado para crianças com desnutrição visa à estabilização hemodinâmica, hidroeletrolítica e acidobásica, seguida pela terapia nutricional propriamente dita ${ }^{9}$.

Por outro lado, no mundo, o número de crianças e adolescentes com obesidade cresceu 10 vezes nos últimos 40 anos. Se essa realidade se mantiver, no Brasil, até 2022, haverá mais crianças e adolescentes com obesidade do que crianças desnutridas. Esse preocupante aumento da obesidade resulta das políticas alimentares que encarecem os produtos nutritivos e saudáveis, dificultando o acesso das comunidades carentes a eles. Apesar do crescimento da obesidade entre crianças no mundo inteiro, a desnutrição moderada ou grave ainda é um grande problema de saúde pública ${ }^{10}$.

A introdução precoce do leite não materno na dieta da criança tem sido um fator que contribui para a obesidade infantil, assim como o aumento do IMC materno e do peso ao nascer. Vale atentar que a introdução precoce de leite não materno, ainda que em aleitamento materno misto, pode diminuir o efeito protetor à obesidade do leite materno no desenvolvimento infantili1.

Em Sobral, um estudo realizado pela Escola de Formação em Saúde da Família Visconde de Sabóia (EFSFVS) observou que $16,7 \%$ dos atendimentos realizados por nutricionistas junto à população infantil, no período entre 2008 e 2010 , ocorreram em virtude de obesidade e $12,5 \%$ devido a sobrepeso, mostrando, assim, alta incidência de obesidade ${ }^{12}$.

\section{A introdução precoce do leite não materno na dieta da criança tem sido um fator que contribui para a obesidade infantil...}

Os períodos críticos para o desenvolvimento de obesidade são, principalmente, o intrauterino, 0 primeiro ano de vida, dos 5 aos 6 anos de idade e a adolescência. Para a abordagem de crianças com obesidade, é imprescindivel identificar os fatores de risco. Além disso, é necessário que as metas em saúde pública priorizem as definições de intervenção, para propiciar a prevenção e o manejo dessa condição. As ações para evitar a obesidade de crianças devem ter início no pré-natal, observando os fatores de risco familiares e a orientação e o cuidado nutricional da gestante ${ }^{13}$.

Nesse sentido, com a tendência dos agravos alimentares entre as crianças, avaliar o estado nutricional de menores de 5 anos de idade viabiliza a adoção de estratégias de saúde que promovam a prevenção e o cuidado de condições relacionadas à má qualidade nutricional.

Diante desse cenário, este estudo teve por objetivo:

- Avaliar o estado nutricional de menores de 5 anos de idade em Sobral, no período entre 2013 e 2017.

\section{METODOLOGIA}

Trata-se de pesquisa documental, descritiva e retrospectiva que analisa uma amostra de dados do estado nutricional de crianças do Município de Sobral, menores de 5 anos de idade, cadastradas no SISVAN/MS no período entre 2013 e 2017. Essas informações estão disponíveis nos relatórios de acompanhamento do SISVAN/MS, no Sistema de Gestão do Bolsa Família (SIGPBF), bem como na estratégia e-SUS Atenção Básica (e-SUS AB).

Sobral se localiza no norte do Ceará, às margens do Rio Acaraú, a $231 \mathrm{~km}$ de distância da capital, Fortaleza. De acordo com a divisão territorial datada de 1993, esse município é constituído por 11 distritos: a) Sobral; b) Aracatiaçu; c) Bonfim; d) 
(aioca; e) (aracará;f) Jaibaras; g) Jordão; h) Rafael Arruda; i) Patriarca; j) São José do Torto; k) e Taperuaba. Sua população é estimada em 206.644 habitantes. Tem clima seco e quente, com temperatura média acima de $26^{\circ} \mathrm{C}^{14}$.

Os relatórios do SISVAN/MS relativos às crianças de ambos os sexos, residentes nas zonas rurais e urbanas do município, foram avaliados por meio dos índices nutricionais: a) peso-para-altura; b) peso-para-idade; c) idade-para-altura; e d) IMC-para-idade. Dados secundários extraídos do SISVAN/MS foram usados para identificar os principais perfis nutricionais e incentivar o desenvolvimento de pesquisas e ações de atenção à saúde junto a crianças com agravos nutricionais.

Os dados foram compilados no programa computacional Microsoft Excel, versão 2010. Neste estudo, as variáveis foram organizadas por ano e por categoria dos índices nutricionais que indicam as crianças com magreza acentuada, sobrepeso e obesidade. 0 programa computacional Microsoft PowerPoint, versão 2010, foi usado para estruturar os gráficos e as tabelas deste artigo.

Por usar informações contidas no SISVAN/MS, com dados de livre acesso disponíveis na internet, este estudo não foi submetido a comitê de ética em pesquisa com seres humanos.

\section{RESULTADOS E DISCUSSÃO}

A Tabela 1 apresenta o índice nutricional calculado pela relação peso $X$ altura ( $\mathrm{X} A)$, usado para identificar tanto o emagrecimento quanto o excesso de peso das crianças. Nesse cálculo, a altura é medida em centímetros, com o indivíduo deitado até os 2 anos e em pé a partir dos 2 anos. 0 peso é registrado em quilos. A massa corpórea (em quilos) é dividida pela altura em centímetros, segundo métodos preconizados pelo Ministério da Saúde (MS), e registrada em gráficos que identificam a faixa de percentil na qual se encontra o indivíduo ${ }^{15}$.

Tabela 1 - Relação peso $X$ altura entre menores de 5 anos de idade em Sobral.

\begin{tabular}{lccccccc}
\hline \multicolumn{1}{c}{ Peso } & $\mathbf{X}$ Altura \\
\hline Ano & $\begin{array}{c}\text { Magreza } \\
\text { acentuada }\end{array}$ & Magreza & Eutrófico & $\begin{array}{c}\text { Risco de } \\
\text { Sobrepeso }\end{array}$ & Sobrepeso & Obesidade & Total \\
\hline 2013 & 119 & 117 & 2.766 & 1.137 & 517 & 629 & 5.285 \\
2014 & 147 & 121 & 3.137 & 1.317 & 627 & 651 & 6.000 \\
\hline 2015 & 152 & 142 & 3.468 & 1.370 & 687 & 630 & 6.449 \\
2016 & 151 & 137 & 3.899 & 1.600 & 801 & 728 & 7.316 \\
\hline 2017 & 138 & 158 & 3.835 & 1.584 & 818 & 814 & 7.347 \\
\hline Total & 707 & 675 & 17.105 & 7.008 & 3.450 & 3.452 & 32.397 \\
\hline
\end{tabular}

Fonte: Elaborada pelos autores.

A Tabela 1 indica evidente predomínio da obesidade e do sobrepeso entre os agravos nutricionais no período estudado. 0 sobrepeso foi o distúrbio nutricional com maior aumento do número de crianças acometidas $(58,2 \%)$. A magreza (35\%) e a magreza acentuada (15\%) também aumentaram - apesar desse crescimento não ser tão acentuado quanto o do sobrepeso e da obesidade, isso deve chamar a atenção dos profissionais da saúde, uma vez que tal fenômeno pode modificar o metabolismo das crianças e levar à redução do ritmo de crescimento. Os casos de desnutrição grave podem interromper o desenvolvimento estatural ${ }^{16}$.

0 aumento do número de crianças obesas em Sobral é compatível com a realidade do país. Na Pesquisa Nacional de Demografia de Saúde da Criança e da Mulher de 2006 (PNDS-2006), o Brasil já apresentava 7\% de crianças obesas e esse percentual se elevou nos últimos anos ${ }^{17}$.

Uma pesquisa realizada em 2014, nas 5 regiões do Brasil, identificou $12 \%$ de crianças obesas. Nesse estudo, a obesidade e o sobrepeso foram relacionados a maiores pesos das crianças ao nascer e, também, a amamentação exclusiva por menor tempo. Essas também são algumas das causas que podem levar ao aumento da obesidade em Sobral. A falta de informação das mães contribui para a redução do tempo de amamentação exclusiva. De acordo com o relato do serviço de enfermagem de um centro da Estratégia Saúde da Família (ESF) nesse município, as mães foram orientadas quanto à importância e à técnica da amamentação no ano de 2011 - isso proporcionou melhores índices de adesão ao aleitamento exclusivo ${ }^{18,19}$. 
A Tabela 2 apresenta a relação altura $X$ idade ( $X$ X I), que expressa o crescimento linear das crianças e corresponde aos dados que melhor demonstram os efeitos adversos em termos de crescimento. Trata-se do índice mais sensivel para aferir a qualidade de vida infantil. A altura é aferida em centímetros e a idade é registrada em meses. Segundo o MS, os gráficos que ilustram esses dados refletem o crescimento de uma população de referência e, a partir deles, pode-se obter a classificação do estado nutricional das crianças, segundo o percentil calculado de altura e idade.

Tabela 2 - Relação altura idade entre menores de 5 anos de idade em Sobral

\begin{tabular}{lcccc}
\hline \multicolumn{5}{c}{ Altura Idade } \\
\hline Ano & $\begin{array}{c}\text { Altura muita baixa } \\
\text { para a idade }\end{array}$ & $\begin{array}{c}\text { Altura baixa para a } \\
\text { idade }\end{array}$ & $\begin{array}{c}\text { Altura adequada para a } \\
\text { idade }\end{array}$ & Total \\
\hline 2013 & 244 & 410 & 4.631 & 5.285 \\
2014 & 293 & 513 & 5.194 & 6.000 \\
2015 & 292 & 530 & 5.627 & 6.449 \\
2016 & 373 & 600 & 6.343 & 7.316 \\
2017 & 406 & 589 & 6.352 & 7.347 \\
Total & $\mathbf{1 . 6 0 8}$ & $\mathbf{2 . 6 4 2}$ & $\mathbf{2 8 . 1 4 7}$ & $\mathbf{3 2 . 3 9 7}$ \\
\hline
\end{tabular}

Fonte: Elaborada pelos autores.

A Tabela 2 indica que, no período estudado, a maior parte das crianças do município apresentou altura adequada para a idade; o número de crianças com altura baixa e muito baixa para a idade cresceu durante praticamente todo o período. Esses números são bastante relevantes, pois, em 2017, identificou-se o maior número de crianças com altura inadequada (13,5\% do total). Um dos fatores relacionados à baixa estatura das crianças é o precário nível econômico de suas famílias, como observado por estudo realizado no Estado da Paraíba em 2016, no qual a ocorrência de baixa estatura foi 2 vezes maior entre crianças com renda per capita familiar menor do que 0,5 salário mínimo em comparação às famílias com renda per capita entre 0,5 e 1 salário mínimo. A baixa estatura e o baixo peso das mães também influenciam a reduzida estatura das crianças. No mesmo estudo, constatou-se que $10 \%$ das crianças de mães com baixa estatura também apresentavam déficit de estatura, ao passo que apenas $5 \%$ das crianças de mães com estatura normal apresentavam baixa estatura. Já em relação às mães com baixo peso, $15 \%$ delas tiveram filhos com baixa estatura, enquanto apenas $6 \%$ das mães com peso normal tiveram filhos com baixa estatura ${ }^{20}$. Outro estudo realizado no Estado da Paraíba detectou que o baixo peso das crianças está fortemente relacionado à idade das mães menores de 20 anos e, ainda, ao baixo peso materno ${ }^{21}$.

$\mathrm{Na}$ Tabela 3 se observa a relação entre a massa corpórea e a idade cronológica das crianças (P X I). Esse índice nutricional é usado, principalmente, para a caracterização do baixo peso e é calculado dividindo a massa corpórea (em quilos) pela idade (em meses), como preconiza o MS. Tal índice está presente na caderneta da criança, distribuída nas maternidades e nos estabelecimentos de saúde ${ }^{15}$.

Tabela 3 - Relação peso X idade entre menores de 5 anos de idade em Sobral

\begin{tabular}{lccccc}
\hline \multicolumn{5}{c}{ Peso $\times$ Idade } \\
\hline Ano & $\begin{array}{c}\text { Peso muito baixo } \\
\text { para a idade }\end{array}$ & $\begin{array}{c}\text { Peso baixo para a } \\
\text { idade }\end{array}$ & $\begin{array}{c}\text { Peso adequado ou } \\
\text { eutrófico }\end{array}$ & $\begin{array}{c}\text { Peso elevado } \\
\text { para a idade }\end{array}$ & Total \\
2013 & 32 & 97 & 4.484 & 672 & 5.285 \\
2014 & 48 & 131 & 5.158 & 663 & 6.000 \\
2015 & 45 & 113 & 5.592 & 699 & 6.449 \\
2016 & 59 & 124 & 6.320 & 813 & 7.316 \\
\hline 2017 & 56 & 177 & 6.300 & 814 & 7.347 \\
\hline Total & 240 & 642 & 27.854 & 3.661 & 32.397 \\
\hline
\end{tabular}

Fonte: Elaborada pelos autores. 
A Tabela 3 aponta que o número de crianças com peso elevado para a idade aumentou no período estudado e manteve-se estável entre 2016 e 2017. 0 maior número de crianças com peso elevado para a idade foi observado em 2017. Em relação ao peso baixo e muito baixo para a idade, os maiores aumentos ocorreram entre 2016 e 2017 - evidenciando que, apesar da transição nutricional no país, Sobral ainda apresenta certo aumento do número de crianças com peso muito baixo e baixo para a idade.

0 baixo peso das crianças pode relacionar-se ao baixo peso materno, como demonstrado por estudo realizado no Estado de Pernambuco, em 2015 - constatou-se que o aumento do peso dos filhos era diretamente proporcional ao aumento do peso materno. A assistência ao pré-natal influencia os bons indicadores nutricionais entre as crianças $^{22}$.

Um estudo realizado em São Paulo, em 2011, observou que mães com baixo peso apresentam quase 3 vezes mais chances de ter seus filhos com peso inadequado ao nascer ${ }^{23}$, corroborando a relação entre o estado nutricional materno e o da criança.

Além disso, vale ressaltar que gestantes adolescentes também apresentam maiores chances de ter filhos com baixo peso, segundo estudo realizado na Bahia, entre 2006 a 2012 - isso demonstra a ligação entre os distúrbios nutricionais neonatais e a idade das mães, pois mães adolescentes são suscetíveis a condições sociais, econômicas e educacionais que podem constituir fatores de risco para as crianças recém-nascidas e aquelas na primeira infância ${ }^{24}$. Pesquisas apontam que fatores como assistência pré-natal inadequada, recusa da gestação pela família ou pelo parceiro ou ausência do parceiro afetam o bem-estar e a saúde da gestante adolescente, contribuindo negativamente para o crescimento e desenvolvimento fetal ${ }^{25}$. Crianças com baixo peso ao nascer demandam atenção, pois apresentam maior risco nutricional no primeiro ano de vida ${ }^{26}$ es riscos de peso inadequado tendem a se manter ao longo da infância.

Na Tabela 4 se observam os dados do IMC $x$ Idade - essa análise é relevante, pois possibilita identificar condições para avaliar o estado nutricional das crianças pelos percentis que relacionam 3 medidas antropométricas (peso, altura e idade). 0 IMC é calculado dividindo a massa corpórea em quilos pela altura em metros elevada ao quadrado. Pode-se classificar o estado nutricional das crianças pelo IMC.

Tabela 4 - Relação IMC x Idade entre menores de 5 anos de idade em Sobral

\begin{tabular}{lccccccc}
\hline \multicolumn{10}{c}{ IMC x Idade } \\
\hline Ano & $\begin{array}{c}\text { Magreza } \\
\text { acentuada }\end{array}$ & Magreza & Eutrofia & $\begin{array}{c}\text { Risco de } \\
\text { sobrepeso }\end{array}$ & Sobrepeso & Obesidade & Total \\
2013 & 166 & 139 & 2.599 & 1.194 & 582 & 605 & 5.285 \\
2014 & 200 & 147 & 2.929 & 1.355 & 730 & 639 & 6.000 \\
\hline 2015 & 195 & 139 & 3.263 & 1.441 & 762 & 649 & 6.449 \\
2016 & 197 & 154 & 3.628 & 1.690 & 919 & 728 & 7.316 \\
\hline 2017 & 169 & 155 & 3.624 & 1.620 & 893 & 886 & 7.347 \\
\hline Total & 927 & 734 & 16.043 & 7.300 & 3.886 & 3.507 & 32.397 \\
\hline
\end{tabular}

Fonte: Elaborada pelos autores.

A Tabela 4 destaca, a partir da relação do IMC $\times$ Idade, o risco de sobrepeso, o sobrepeso e a obesidade como agravos entre as crianças nesse período. 0 risco de sobrepeso cresceu no período de 2013 a 2016 , porém, de 2016 a 2017, esse número diminuiu 4,1\%. 0 número de crianças com sobrepeso também aumentou no período de 2013 a 2016; já no período de 2016 a 2017 houve redução de 2,8\%. Vale salientar que o número de crianças obesas cresceu durante todo o período estudado: de 2013 a 2017 houve aumento de $46 \%$ do número de crianças com obesidade. 0 aumento dos índices de obesidade em Sobral não difere dos resultados de outros municípios do Brasil, como Campina Grande, que também apresenta crescimento desse distúrbio nutricional entre crianças, consequência do maior consumo de alimentos ricos em açúcar, como doces, salgadinhos e refrigerantes. A substituição do café da manhã e de outras refeições por lanches ao longo do dia também se apresenta como fator de risco para a obesidade ${ }^{20}$.

Um estudo realizado em Minas Gerais relacionou a alimentação inadequada das crianças à baixa ingestão de frutas, verduras e leguminosas e aos altos índices de obesidade detectados entre escolares nesse estado 
no ano de $2015^{27}$.

A má qualidade alimentar também é uma das causas do aumento dos índices de obesidade em Sobral. Nesse município, as refeições das crianças incluem alimentos ricos em gorduras e açúcares, como pizzas, refrigerantes e salgadinhos ${ }^{28}$.

A ausência de atividade física contribui para 0 aumento do número de crianças obesas, pois estudos apontam que essa prática assume papéis na prevenção e no tratamento do sobrepeso e da obesidade: a) restaura o perfil lipídico; b) melhora a composição corpórea; e c) restaura o metabolismo adequado ${ }^{29}$.

\section{CONCLUSÃO}

Este estudo analisou a transição nutricional que o Município de Sobral vem enfrentando, caracterizada por diversos estados nutricionais em menores de 5 anos de idade, adotando o SISVAN/MS como fonte de dados no período entre 2013 e 2017.

Os resultados mostram que o número de crianças acometidas por magreza e magreza acentuada, além de altura baixa e altura muito baixa para a idade, cresceu no município. 0s índices de peso baixo e peso muito baixo para a idade também aumentaram na população estudada. Contudo, o agravo nutricional com crescimento mais acentuado foi o sobrepeso e a obesidade.

Diante da coexistência das diversas condições relacionadas à alimentação em menores de 5 anos de idade, conclui-se que Sobral passa por um processo de transição nutricional. Urge, assim, a necessidade de implementar ações na APS, além de investir em pesquisas voltadas a reduzir e corrigir as carências nutricionais, promovendo hábitos saudáveis desde a mais tenra infância.

\section{CONTRIBUIÇÃO DOS AUTORES}

Beatriz Mendes Alves contribuiu com a realização da pesquisa, o delineamento do estudo e a redação do manuscrito. João Vitor Souza Marques contribuiu com a realização da pesquisa e a redação do manuscrito. Cynara Carvalho Parente contribuiu com a redação e revisão crítica do manuscrito. Marcos Vinicius Souza Marques contribuiu com a realização da pesquisa. Francisco Plácido Nogueira Arcanjo e Karyne Gomes Cajazeiras contribuíram com a revisão crítica do manuscrito.

\section{REFERÊNCIAS}

1. Pereira IFS. Estado nutricional em menores de 5 anos de idade no Brasil: evidências de polarização epidemiológica nutricional. Ciênc Saúde Colet [serial on the internet]. 2017 [cited 2018 Nov 19];22(10):3341-52. Available from: http://www.scielo.br/pdf/csc/v22n10/1413-8123csc-22-10-3341.pdf

2. Brasil. Política Nacional de Alimentação e Nutrição. Brasília (DF): Ministério da Saúde; 2012.

3. Sistema de Vigilância Alimentar e Nutricional [homepage on the internet]. c2004 [cited 2018 Nov 27]. Available from: http://dab.saude. gov.br/portaldab/ape vigilancia alimentar. php?conteudo=sisvan

4. Sarni R0, Munekata RV. Terapia nutricional na desnutrição energético-proteica grave. In: Lopez FA, Sigulem DM, Taddei JA, editors. Fundamentos da terapia nutricional em pediatria. São Paulo: Sarvier; 2002. p. 115-32.

5. Araújo ST, Oliveira CSM, Muniz PT, Nunes MS, Cardoso MA. Desnutrição infantil em um dos municípios de maior risco nutricional do Brasil: estudo de base populacional na Amazônia ocidental brasileira. Rev Bras Epidemiol [serial on the internet]. 2016 [cited 2018 Nov 19];19(3):554-66. Available from: http://www.scielo.br/pdf/rbepid/ v19n3/1980-5497-rbepid-19-03-00554.pdf

6. Oliveira ACM, Pereira LA, Ferreira RC, Clemente APG. Estado nutricional materno e sua associação com o peso ao nascer em gestações de alto risco. Ciênc Saúde Colet [serial on the internet]. 2018 [cited 2018 Nov 19];23(7):2373-82. Available from: http://www.scielo.br/pdf/csc/v23n7/1413-8123csc-23-07-2373.pdf

7. Organización de las Naciones Unidas para la Alimentación y la Agricultura. El estado de la seguridad alimentaria y la nutrición en el mundo. Roma: FA0; 2018.

8. Sucupira ACSL, Andrade LOM, Barreto ICHC, Lima JW, Santiago AV, Santiago AX. Determinantes sociais da saúde de crianças de 5 a 9 anos da zona urbana de Sobral, Ceará, Brasil. Rev Bras Epidemiol [serial on the internet]. 2018 [cited 2018 Nov 26];(17):160-77. Available from: http://www.scielo.br/pdf/rbepid/ v17s2/pt 1415-790X-rbepid-17-s2-00160.pdf

9. Brasil. Manual de terapia nutricional na atenção especializada hospitalar. Brasília (DF): Ministério da Saúde; 2016. 
10. NCD Risk Factor Collaboration. Worldwide trends in body-mass index, underweight, overweight, and obesity from 1975 to 2016: a pooled analysis of 2416 population-based measurement studies in 128.9 million children, adolescents, and adults. J Lancet [serial on the internet]. 2017 [cited 2018 Nov 27];(390):2627-42. Available from: https://www.thelancet.com/action/showPdf?pii $=$ S0140-6736\%2817\%2932129-3

11. Nascimento VG. Aleitamento materno, introdução precoce de leite não materno e excesso de peso na idade pré-escolar. Rev Paul Pediatr [serial on the internet]. 2016 [cited 2018 Nov 29];34(4):4549. Available from: http://www.scielo.br/pdf/rpp/ v34n4/pt 0103-0582-rpp-34-04-0454.pdf

12. Barbosa MIS. O nutricionista na Estratégia de Saúde da Família. Rio de Janeiro: Ed. UERJ; 2016.

13. Brasil. Manual de diretrizes para o enfretamento da obesidade na saúde suplementar brasileira. Rio de Janeiro: Agência Nacional de Saúde Suplementar; 2017.

14. Instituto Brasileiro de Geografia e Estatística [homepage on the internet]. Brasil/Ceará: Sobral. c2019 [cited 2018 Dec 2]. Available from: https://cidades.ibge.gov.br/brasil/ce/sobral/ pesquisa $/ 32 / 28163$

15. Brasil. SISVAN: notas técnicas [document on the internet]. 2004 [cited $2018 \mathrm{Dec} 4$ ]. Available from: http://tabnet.datasus.gov.br/cgi/SISVAN/CNV/ notas sisvan.html

16. Alecrim JS, Sousa LF, Castro JM, Borja GPC, Castro LLB, Bastos $V C$, et al. Prevalência de obesidade infantil em uma escola pública da Cidade de Ipatinga (MG). Ensaios e Ciência: Biológicas, Agrárias e da Saúde [serial on the internet]. 2018 [cited 2018 Dec 5];22(1):22-6. Available from: http://pgsskroton. com.br/seer/index.php/ensaioeciencia/article/ view/4501/4267

17. Monteiro CA, Conde WL, Konno SC, Lima AL, Silva $A C$, Benicio MH. Avaliação antropométrica do estado nutricional de mulheres em idade fértil e crianças menores de cinco anos. In: Brasil. Pesquisa Nacional de Demografia e Saúde da Criança e da Mulher - PNDS 2006: dimensões do processo reprodutivo e da saúde da criança. Brasília (DF): Ministério da Saúde; 2009. p. 213-30.

18. Muller MR, Tomasi E, Facchini LA, Piccini RX, Silveira DS, Siqueira FV, et al. Excesso de peso e fatores asociados em menores de 5 anos em populações Urbanas no Brasil. Rev Bras Epidemiol [serial on the internet]. 2014 [cited 2018 Dec 5];17(2):285-96. Available from: http://www.scielo.br/pdf/rbepid/ v17n2/pt 1415-790X-rbepid-17-02-00285.pdf
19. Martins QPM. Fatores que influenciam no aleitamento materno exclusivo: um relato de experiência. II Congresso Brasileiro de Enfermagem Neonatal; 2012; Fortaleza, BR [document on the internet]. 2012 [cited 2018 Dec 6]. Available from: http://www.abenfoce.org.br/sites/default/ files/FATORES $\% 20$ QUE $\% 2$ OINFLUENCIAM $\% 20$ N0\%20 ALEITAMEN T $0 \% 20$ MATERN $0 \% 20$ EXCLUSIV $0 \% 20$ $\underline{U M \% 20} 0 . p \mathrm{df}$

20. Pedraza DF, Sales MC, Menezes TN. Fatores associados ao crescimento linear de crianças socialmente vulneráveis do Estado da Paraíba, Brasil. Ciênc Saúde Colet [serial on the internet]. 2016 [cited 2018 Dec 6];21(3):935-45. Available from: http://www.scielo.br/pdf/csc/v21n3/14138123-csc-21-03-0935.pdf

21. Souza MM, Pedraza DF, Menezes TN. E s t a d o nutricional de crianças assistidas em creches e situação de (in)segurança alimentar de suas famílias. Ciênc Saúde Colet [serial on the internet]. 2012 [cited 2018 Dec 6];17(12):3425-36. Available from: https://www.scielosp.org/pdf/csc/2012. v17n12/3425-3436

22. Miglioli TC. Fatores associados ao estado nutricional de crianças menores de cinco anos. Rev Saúde Pública [serial on the internet]. 2015 [cited 2018 Dec 6];(49):[about 8 pages]. Available from: http://www.scielo.br/pdf/rsp/v49/pt 0034-8910rsp-S0034-89102015049005441.pdf

23. Fonseca MRCC, Laurenti R, Marin CR, Traldi MC. Ganho de peso gestacional e peso ao nascer do concepto: estudo transversal na região de Jundiaí, São Paulo, Brasil. Ciênc Saúde Colet [serial on the internet]. 2014 [cited 2018 Dec 6];19(5):1401-7. Available from: https://www.scielosp.org/article/ ssm/content/raw/?resource ssm path $=/ \mathrm{media} /$ assets/csc/v19n5/1413-8123-csc-19-05-01401.pdf

24. Santos NLAC, Costa MCO, Amaral MTR, Vieira G0, Bacelar EB, Almeida AHV. Gravidez na adolescência: análise dos fatores de risco para baixo peso, prematuridade e cesariana. Ciênc Saúde Colet [serial on the internet]. 2014 [cited 2018 Dec 6];19(3):71926. Available from: http://www.scielo.br/scielo. php?pid =S1413-81232014000300719\&script=sci abstract $\& \mathrm{t} \operatorname{lng}=p t$

25. Chen XK, Wen SW, Fleming N, Demissie K, Rhoads GG, Walker M. Teenage pregnancy and adverse birth outcomes: a large population based retrospective cohort study. Int J Epidemiol. 2007;36(2):368-73.

26. Motta MEFA, Silva GAP, Araújo OC, Lira PI, Lima MC. 0 peso ao nascer influencia o estado nutricional ao final do primeiro ano de vida? J Pediatr (Rio J) [serial on the internet]. 2005 [cited 2018 Dec 6];81(5):377-82. Available from: http://www.scielo. $\mathrm{br} / \mathrm{pdf} / \% 0 \mathrm{D} / \mathrm{jped} / \mathrm{v} 81 \mathrm{n} 5 / \mathrm{v} 81 \mathrm{n} 5 \mathrm{a} 07 . \mathrm{pdf}$ 
27. Paiva ACT, Couto CC, Masson APL, Monteiro CAS, Freitas CF. Obesidade infantil: análises antropométricas, bioquímicas, alimentares e estilo de vida. Rev Cuid (Bucaramanga 2010) [serial on the internet]. 2018 [cited 2018 Dec 6];9(3):113. Available from: https://www.researchgate. net/publication/327494328 0besidade Infantil analises antropometricas bioquimicas alimentares e estilo de vida

28. Lira RCM, Antunes LR, Mesquita K0. Experiência de abordagem sobre alimentação entre crianças. Sanare (Sobral, Online) [serial on the internet]. 2016 [cited 2018 Dec 7];15(1):69-73. Available from: https://sanare.emnuvens.com.br/sanare/article/ viewFile/930/559

29. Paes ST, Marins JCB, Andreazzi AE. Efeitos metabólicos do exercício físico na obesidade infantil: uma visão atual. Rev Paul Pediatr [serial on the internet]. 2015 [cited 2018 Dec 7];33(1):1229. Available from: http://www.scielo.br/pdf/rpp/ v33n1/pt 0103-0582-rpp-33-01-00122.pdf
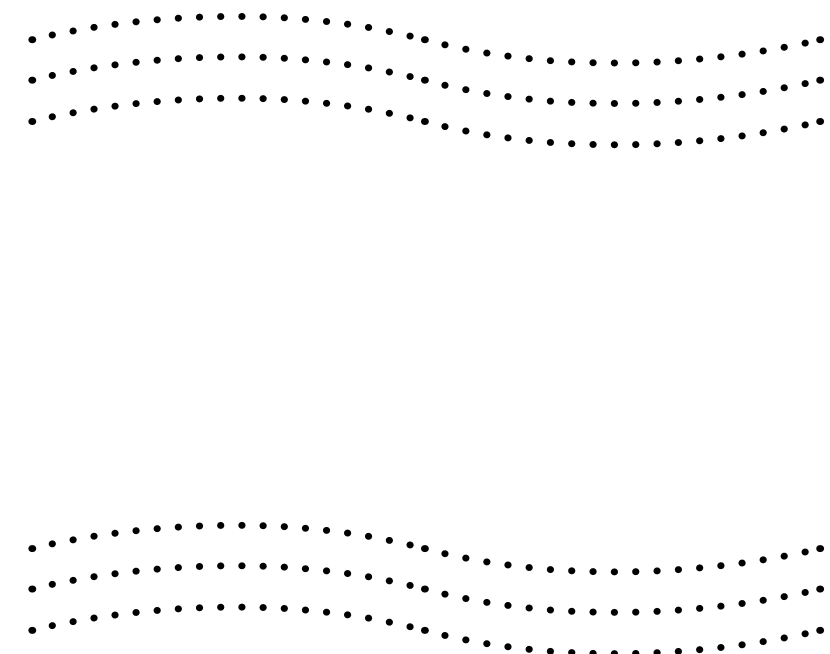
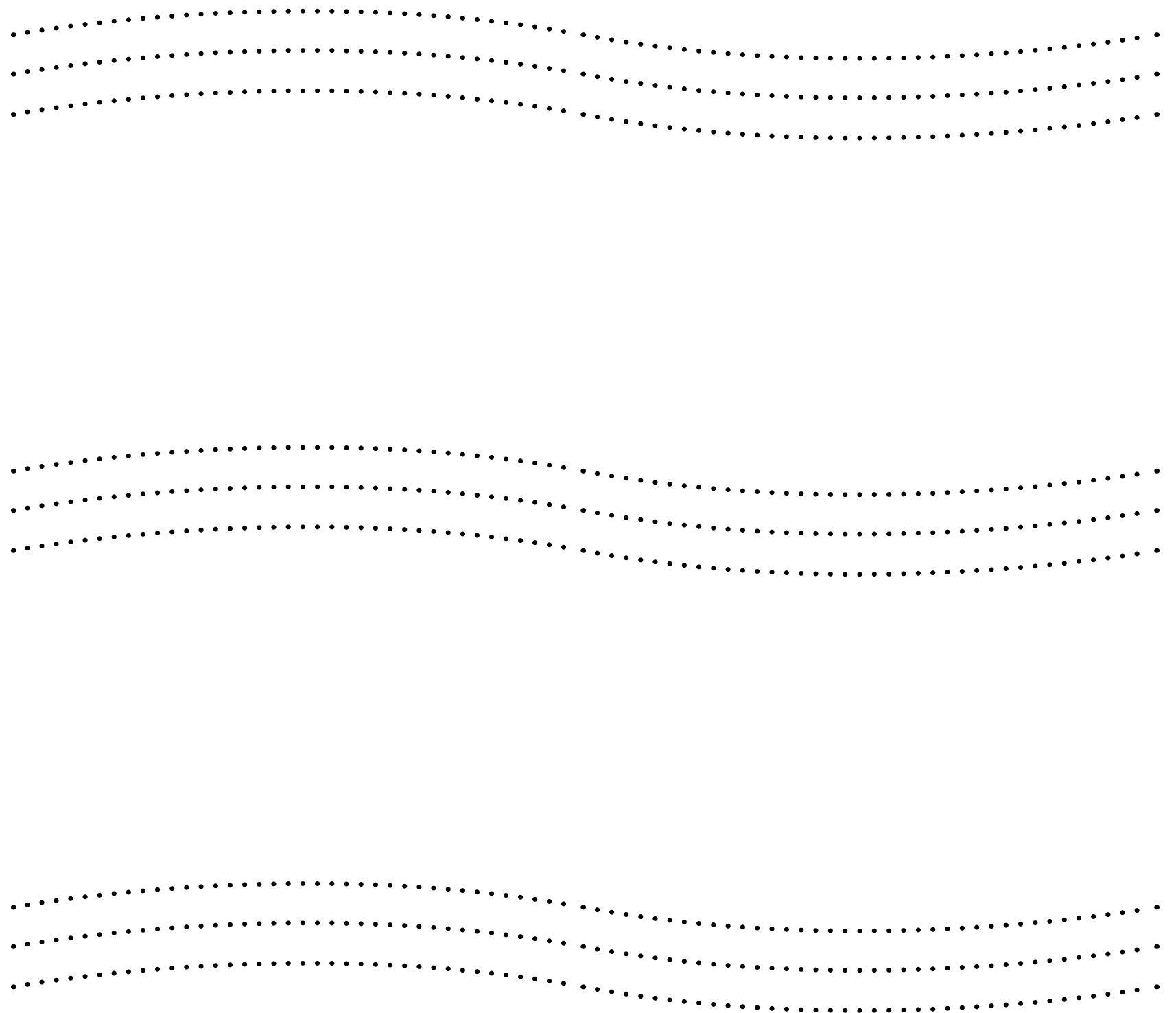